\title{
COVID detection from chest X-ray using MobileNet and Residual Separable Convolution block
}

\author{
Jagadeesh Kakarla* • Isunuri Bala Venkateswarlu
}

Received: date / Accepted: date

\begin{abstract}
A newly emerged coronavirus disease affects the social and economical life of the world. This virus mainly infects the respiratory system and spreads with airborne communication. Several countries witness the serious consequences of the COVID pandemic. Early detection of COVID infection is the critical step to survive a patient from death. The chest radiography examination is the fast and cost-effective way for COVID detection. Several researchers have been motivated to automate COVID detection and diagnosis process using chest X-ray images. However, existing models employ deep networks and are suffering from high training time. This work presents transfer learning and residual separable convolution block for COVID detection. The proposed model utilizes pre-trained MobileNet for binary image classification. The proposed residual separable convolution block has improved the performance of basic MobileNet. Two publicly available datasets COVID5K, and COVIDRD have considered for the evaluation of the proposed model. Our proposed model exhibits superior performance than existing state-of-art and pretrained models with $99 \%$ accuracy on both datasets. We have achieved similar performance on noisy datasets. Moreover, the proposed model outperforms existing pretrained models with less training time and competitive performance than basic MobileNet. Further, our model is suitable for mobile applications as it uses fewer parameters and lesser training time.
\end{abstract}

Jagadeesh Kakarla

IIITDM Kacheepuram, Chennai, India

E-mail: jagadeeshk@iiitdm.ac.in

\section{Introduction}

The newly discovered severe acute respiratory syndrome coronavirus 2 (SARS-CoV-2) has triggered the latest outbreak namely coronavirus disease (COVID) [1]. The epidemic disease has affected the social and economical life of the world and has spread rapidly within few months. Several countries witness the serious consequences of the COVID pandemic. Recently, COVID has reiterated in few countries as a second wave with incremental growth. World Health Organization (WHO) has reported that globally 161,513,458 confirmed cases of COVID, including 3,352,109 deaths till April 2021 [2]. Thus, COVID detection and diagnosis has received contemporary research task.

The COVID disease is a type of pneumonia that infects the respiratory system and spreads through close contact. The isolation of infected patients is the preliminary step to break communal spread. In addition to that appropriate medication can increase the survival of patients from death. The detection of COVID infection becomes a tedious task from the physical symptoms like fever, cold, dyspnea, fatigue, and myalgia [3]. The reverse transcription-polymerase chain reaction (RT$\mathrm{PCR}$ ) is a traditional clinical test used for the detection of COVID infection. However, long turnaround time and limited availability of testing kits are the major difficulties with RT-PCR tests[4].

Nowadays, the medical imaging plays vital role in healthcare services for disease detection [5]. Some of the health care applications are brain metastases detection [6], ischemic stroke detection [7], ulcer classification [8], and patient risk prediction [9]. Recent studies has proved that, the medical imaging technology can be an alternative to RT-PCR as it is highly sensitive for the diagnosis and screening of COVID [10] [4]. Apart 
from the clinical tests, radiography examination including $\mathrm{CT}$ and X-ray is the fast and cost-effective test for COVID detection. Moreover, digital X-ray equipment is available in most hospitals and no need for any additional transportation cost. In general, COVID infection can be identified by the examination of multifocal and bilateral ground-glass opacity and/or consolidation [3][11]. Ground glass opacity is referred to as a region of hazy lung radiopacity in chest radiography. The central mediastinum and heart appear as white in a normal chest X-ray and the lungs appear in black due to air. There is a change in blackness at the lung portion due to denser ground-glass opacity in COIVD infection. Fig. 1 (a) depicts normal chest X-ray finding while Fig. 1 (b) visualizes ground-glass opacity (white arrows) due to COVID infection. Similarly, outlined arrows in Fig. 1 (b) indicates a consolidation of left upper and mid zones of lungs. Thus, the identification of ground-glass opacity and consolidation patterns is essential for COVID detection.

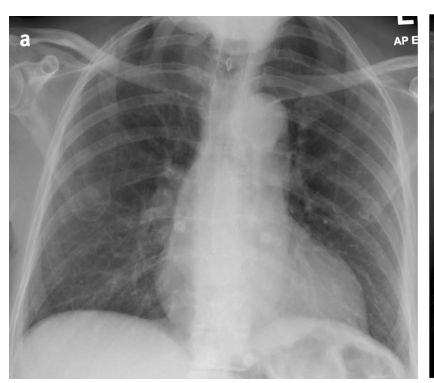

(a) Normal finding

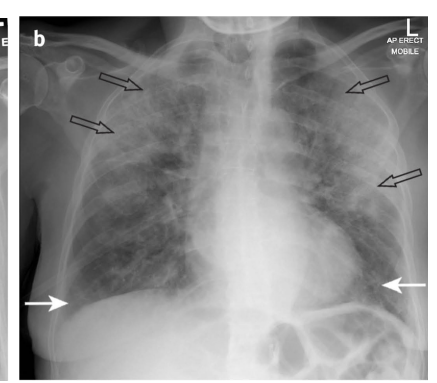

(b) COVID infection
Fig. 1: Chest X-ray for COVID detection

Visual examination of these patterns is a challenging task for computer-aided COVID detection systems. The deep learning models are successful for such visual pattern analysis and classification. In this regard, several proposals on COVID detection from chest X-ray images have been reported and some of the noticeable models are as follows. Afshar et al. [12] have implemented a framework known as COVID-CAPS which is based on a capsule network for COVID detection. Authors have achieved an accuracy of $95.7 \%$ and $98.3 \%$ with COVID-CAPS without pre-training and with pretraining respectively. Sakib et al. [13] have generated synthetic chest X-ray images with COVID infection to train the custom CNN model using generic data augmentation and generative adversarial network. Authors have achieved $93.94 \%$ of accuracy in COVID detection. Abbas et al. [14] have proposed a DeTraC model that investigates class boundaries using a class decom- position mechanism. An accuracy of $93.1 \%$ has been achieved for COVID detection from X-Ray images. Minaee et al. [15] have presented Deep-COVID using deep transfer learning for prediction of COVID and reported a sensitivity rate of $98 \%$.

Horry et al. [16] have optimized the VGG19 model for COVID detection from X-Ray, Ultrasound, and CT scan images. They have attained $86 \%, 100 \%$, and $84 \%$ of precision on X-Ray, Ultrasound, and CT scans respectively. Moura et al. [17] have designed automatic approaches for chest X-ray classification in three cases. An accuracy of $79.62 \%, 90.27 \%$, and $79.86 \%$ have been reported in three considered cases respectively. Existing proposals have devised deep neural networks to achieve better accuracy. However, an effective COVID detection system implementation is still a challenging task due to the recent spreading trend of the COVID [13]. Moreover, the implementation of time-efficient models with better performance is still challenging.

Apart from the implementation issues, the class imbalance is one of the significant drawbacks of existing COVID datasets. An insufficient number of COVID positive samples and progressive updates of datasets are major concerns about COVID datasets. We have considered two publicly available chest X-ray datasets for COVID detection. The datasets have been chosen such that one dataset consists of data in balance and the other dataset has balanced data. Details of the two datasets are as follows.

1. COVID-XRay-5K (COVID5K) has created by Minaee et al.[15] and has recently updated with 5184 chest X-ray images. This dataset can be used for binary classification as it contains 5000 normal chest X-ray images and 184 COVID positive images. This dataset exhibits data in balance with a huge number of COVID negative chest X-ray images.

2. Covid radiography (COVIDRD) database of chest X-ray images has recently published by Kaggle [18]. This dataset consists of three classes including normal (1341), COVID (1200), and viral pneumonia (1345). As our objective is COVID detection and hence we have considered only normal and COVID positive chest X-ray images. This dataset presents the balanced positive and negative chest X-ray images.

The COVID detection from chest X-ray images has become contemporary research due to implementation and dataset issues. This motivated us to implement a time-efficient COVID detection model that can work on both datasets. We have proposed a transfer learning and residual separable convolution block model for bi- 
nary X-ray image classification. Major contributions of the work are as follows.

1. Implementation of time-efficient generalized model for COVID detection on different COVID datasets.

2. The proposed residual separable convolution block improves the performance of the basic MobileNet model.

3. Our proposed model is compatible with Mobile vision application as it uses MobileNet and produces similar performance with reduced input sizes.

The remaining paper has organized as follows; Section 2 elaborates details of the proposed methodology. The quantitative analysis of the proposed model has discussed in Section 3 and Section 4 presents the conclusions of the paper.

\section{Proposed methodology}

Recently, deep neural networks have been established as successful hands-on models for image classification due to the availability of a huge Imagenet dataset. MobileNet, ResNet, GoogleNet, and Inception ResNet are the most popular models for classification. However, there is a scarcity of large medical image datasets like Imagenet for medical image classification. It motivated researchers to employ transfer learning to improve medical image classification performance. In this section, we have presented the transfer learning procedure along with implementation details of the proposed model.

\subsection{Transfer learning}

Transfer learning involves sharing of weights or knowledge extracted from one problem domain to solve other related problems. High accuracy can be achieved with transfer learning when problems are closely related. The existing pre-trained models are trained with the Imagenet dataset and can perform thousand of class classification. It needs customization of the output layer to handle binary classification. Fig. 2 (a) visualizes a pre-trained model for COVID detection and the implementation steps are as follows.

1. Firstly, a pre-trained model is assigned with Imagenet weights and then output layers are removed to customize the output layer.

2. Multi-dimensional feature map obtained from the above pre-trained model is then flattened to generate a one-dimensional feature vector.

3. A softmax layer with two neurons is employed to produce the final classification results from the above one-dimensional feature vector.
The pre-trained model obtained from the above process needs training with medical image data. Thus, the models are trained with chest X-ray image datasets for the detection of COVID infection.

\subsection{Proposed residual separable convolution block}

In our experiments, we have observed that GoogleNet performs well on both datasets. On the other hand, Inception ResNet exhibits the worst performance on the COVIDRD dataset due to the insufficient size of the dataset. However, MobileNet and ResNet50 produce average performance due to ample convolutions. MobileNet [19] is the popular time-efficient model designed especially for mobile vision applications. Our objective is to design a fast COVID detection and hence we have considered pre-trained MobileNet. We have proposed a residual separable convolution (RSC) block as shown in Fig. 2 (b) to improve MobileNet performance. In this process, we have replaced flatten layer of the pre-trained MobileNet model with an RSC block. The resulting model is referred to as MobileNet and residual separable convolution block (MNRSC) model. The proposed RSC block uses two separable convolution layers, a global average pooling, a dense layer, and a dropout layer to enhance the spatial feature vectors. We have devised separable convolution with a factored residual connections to reiterate feature maps. Then a global pooling layer converts the multi-dimensional spatial features into a one-dimensional feature vector. In general, the global pooling acts as flatten layer and also avoids over-fitting [20]. A dense layer with 512 neurons has been employed to establish a traditional neural network. In addition to global average pooling, we have utilized a dropout layer after dense layer to further reduce over-fitting due to class imbalance. The proposed MNRSC model has been designed using MobileNet and an RSC block. The steps involved in the implementation of MNRSC are as follows.

\section{Pre-trained MobileNet:}

It accepts input image $\mathbf{I}(w, h, c)$ of size $(224 \times 224 \times 3)$ and produces feature vector $\mathbf{M N}(x, y, z)$ of size $(7 \times 7 \times 1024)$.

2. RSC block: It transforms $\mathbf{M N}(x, y, z)$ into vector $\mathbf{F}(x)$ as follows.

- Two successive separable convolutions $(S C 2 D)$ with 384 filters having kernel size of $(1 \times 1)$ are performed to generate feature maps of $\mathbf{S C 1}(x, y, z)$ and $\mathbf{S C 2}(x, y, z)$ having dimensions as $(7 \times 7 \times 256)$.

$\mathbf{S C 1}(x, y, z)=S C 2 D(\mathbf{M N}(x, y, z))$
$\mathbf{S C 2}(x, y, z)=S C 2 D(\mathbf{S C 1}(x, y, z))$ 


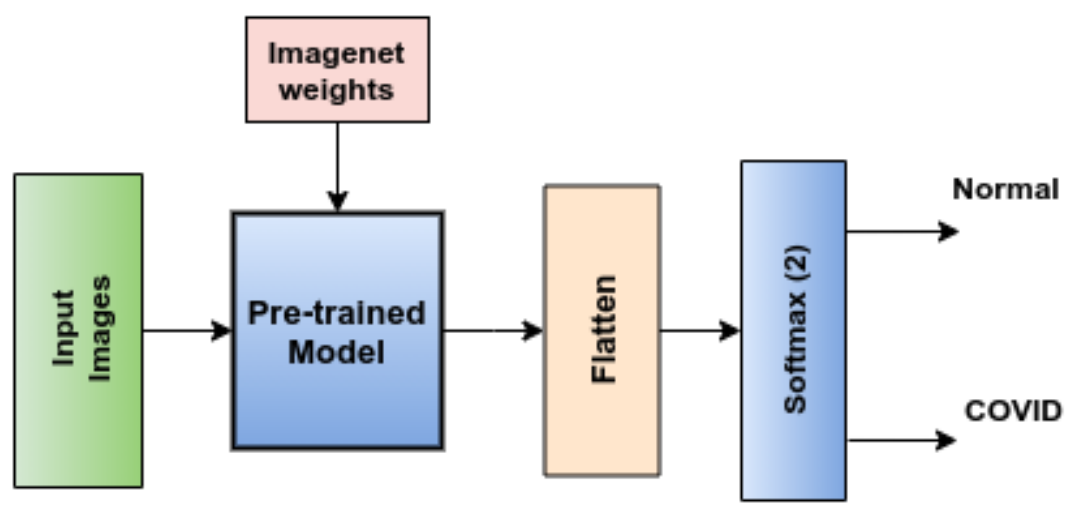

(a) Pre-trained model

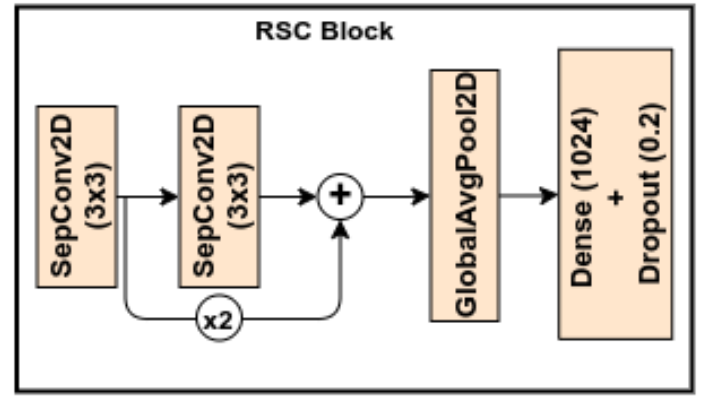

(b) Proposed model

Fig. 2: Block diagram of the proposed model

- Now, factored $\mathbf{S C 1}(x, y, z)$ is added to $\mathbf{S C 2}(x, y, z) \quad 3.1$ Experimental setup to produce feature vector of size $(7 \times 7 \times 512)$. This step invokes factored residual connection to avoid degradation problem of neurons.

$$
\operatorname{SC2}(x, y, z)=\operatorname{SC2}(x, y, z)+2 * \operatorname{SC1}(x, y, z)
$$

- Global average pooling has been employed to generate feature vector $\mathbf{G}(k)$ of size 512 . It maps a three-dimensional vector into a one-dimensional vector.

$$
\mathbf{G}(z)=\frac{\sum_{j=1}^{y} \sum_{i=1}^{x} \mathbf{S C 2}(i, j, z)}{x * y}
$$

- Finally, a dense layer with 512 neurons forms a fully connected neural network to produce feature vector $\mathbf{F}(z)$ of size 512 .

$$
\mathbf{F}(z)=\operatorname{Dense}(\mathbf{G}(z))
$$

\section{Classification:}

Finally, a softmax layer with two neurons has been utilized for binary chest X-ray classification as Normal or COVID.

\section{Results and discussion}

In this section, we have presented a detailed analysis of the proposed model using various parameters including input size, and noisy dataset. Four popular finetuned models including MobileNet, InceptionResNet, GoogleNet, and ResNet have been considered for the evaluation. The proposed model has been compared with the recent COVID detection model i.e. Deep-COVID proposed by Minaee et al. [15].

We have evaluated the proposed model using four vital performance metrics like accuracy, sensitivity, specificity, and jaccard similarity. Sensitivity measures the true positive rate while specificity calculates the true negative rate. Accuracy and jaccard similarity focus on overall classification performance. The proposed model and other pre-trained models have implemented using python and tensorflow. All experiments have been conducted on an Intel Xeon processor with a 25 GB GPU system. We have trained the proposed and pre-trained models using Adam optimizer with an initial learning rate of 0.0001 . Table 1 lists out the complete hyperparameter setup utilized for the experiments.

Table 1: Hyperparameter setup

\begin{tabular}{|c|c|}
\hline Hyperparameter & Value \\
\hline Batch size & 8 \\
Optimizer & Adam \\
Initial learning rate & 0.0001 \\
Number of epochs & 10 \\
\hline
\end{tabular}

\subsection{Evaluation on COVID5K dataset}

COVID-Xray-5K (COVID5K) dataset is the publicly available dataset published by Minaee et al.[15]. It consists of 5184 chest X-ray images. The proposed model has evaluated on the dataset and Table 2 lists out crossvalidation results. The proposed MNRSC model has achieved $100 \%$ accuracy in the first fold and 99\% in other folds. Similar performance has observed in other metrics. This table also manifest that the proposed model procures a mean accuracy of $99 \%$ with a standard deviation of $0.2 \%$. Fig. 3 depicts training and validation 
loss of the proposed MNRSC model in the fourth fold. The proposed MNRSC model exhibits a high validation loss in the first three folds and attains fast convergence within ten epochs due to use of Adam optimizer. Fig. 3 demonstrates that the validation loss becomes consistent after the sixth fold. The proposed model achieves consistent loss due to the deployment of a residual separable convolution block.

Table 2: Fivefold cross-validation on COVID5K dataset

\begin{tabular}{|c|cccc|}
\hline Fold & Accuracy & Sensitivity & Specificity & Jaccard \\
\hline 1 & 100.00 & 100.00 & 100.00 & 100.00 \\
2 & 99.86 & 99.86 & 99.85 & 99.72 \\
3 & 99.52 & 99.52 & 99.65 & 99.08 \\
4 & 99.66 & 99.66 & 99.80 & 99.34 \\
5 & 99.61 & 99.61 & 99.60 & 99.27 \\
\hline Avg. & 99.73 & 99.73 & 99.78 & 99.48 \\
Std. & 0.20 & 0.20 & 0.16 & 0.37 \\
\hline
\end{tabular}

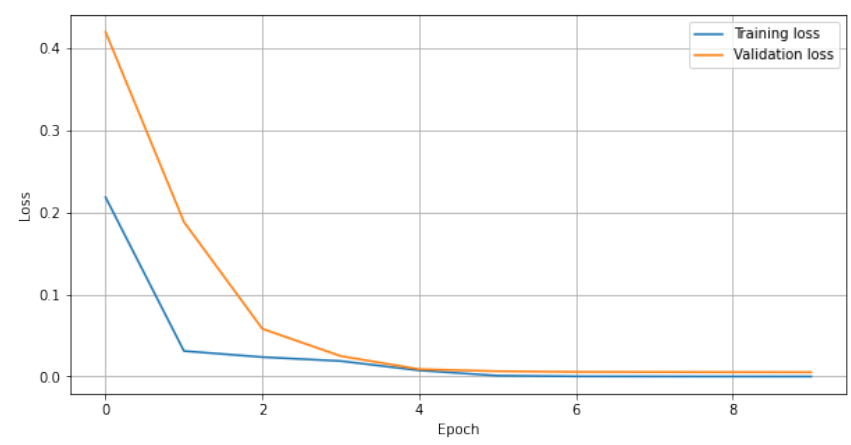

Fig. 3: Training and validation loss on COVID5K dataset

Fig. 4 visualizes the confusion matrix obtained by the proposed model on COVID5K dataset. From this figure, it can be observed that only two COVID positive samples have been wrongly classified. Whereas, six COVID negative samples are classified wrongly on the COVID5K dataset. We have investigated the reasons behind the behavior of the proposed model. Table 3 lists out three X-ray image samples from COVID5K dataset. We have found that our proposed model is ineffective with dis-oriented images and can be observed from samples (3) of Table 3. In this samples, other parts like the abdomen have been included due to wrong orientation. However, our model classifies correctly in case of other images.

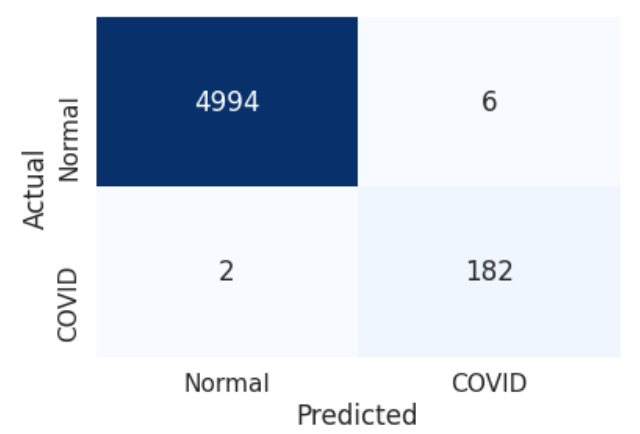

Fig. 4: Confusion matrix on COVID5K dataset

Table 3: Sample X-ray images along with actual and predicted labels

\begin{tabular}{|c|c|c|c|}
\hline & \multicolumn{3}{|c|}{ COVID5K } \\
\hline & (1) & $(2)$ & (3) \\
\hline Actual & 0 & 1 & 0 \\
\hline Predicted & 0 & 1 & 1 \\
\hline
\end{tabular}

\subsubsection{Comparison with state-of-art model}

The proposed model has been compared with the recent COVID detection model namely Deep-COVID proposed by Minaee et al. [15]. Authors have reported sensitivity and specificity and hence we also have considered the same metrics for comparison. Table 4 lists out the performance comparison with the Deep-COVID model. The Deep-COVID model attains 98\%, and $92.9 \%$ of sensitivity, and specificity respectively. The proposed MNRSC model outperforms the existing model with $99 \%$ of sensitivity and specificity. It proves that our model achieves an improvement of $1 \%$ and $6 \%$ in sensitivity and specificity respectively.

Table 4: Comparison with state-of-art model

\begin{tabular}{|c|cc|}
\hline Model & Sensitivity & Specificity \\
\hline Deep-COVID & 98.00 & 92.90 \\
MNRSC & 99.73 & 99.78 \\
\hline
\end{tabular}

\subsubsection{Comparison with pre-trained models}

We have identified four best-performing pre-trained models including GoogleNet, Inception ResNet, MobileNet, and ResNet50, whose accuracy is greater than $98 \%$. These models have produced similar performance on 
Table 5: Sample noise image generation
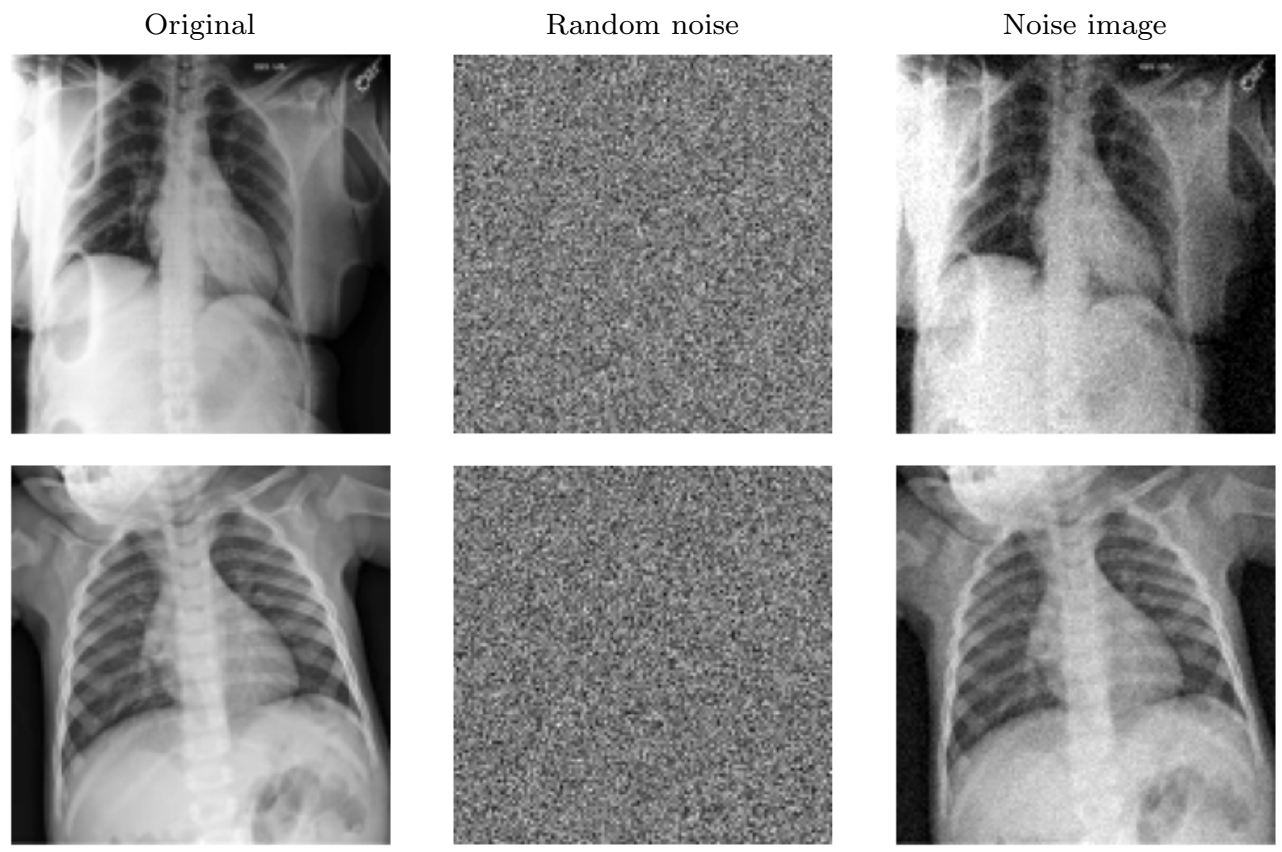

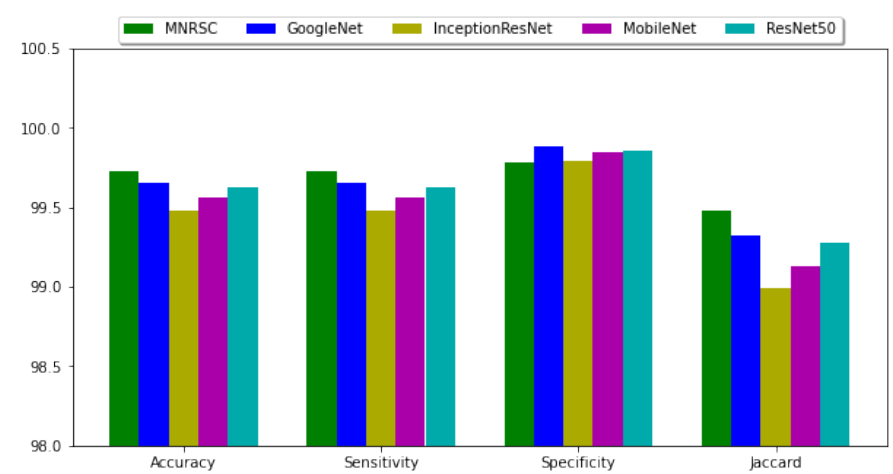

(a) input size $(224 \times 224)$

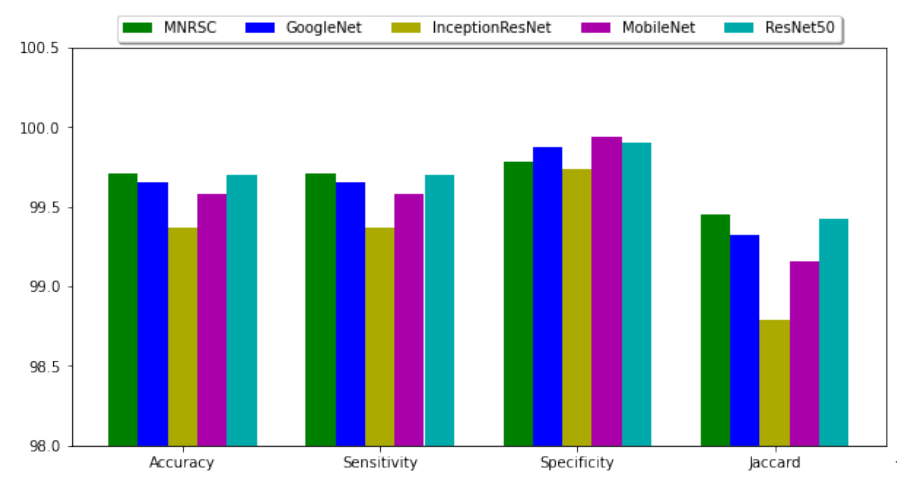

(b) input size $(128 \times 128)$

Fig. 5: Performance comparison on COVID5K dataset

both datasets after fine-tuning. The input size is the pri- mary factor that influences the computational cost and performance. The low-resolution images are preferred for fast computation especially for time-constrained applications like mobile applications. Thus, we have started our experiments with various input sizes including $(224 \times 224)$ and $(128 \times 128)$ for the evaluation. The detailed analysis with various input sizes is as follows.

- Performance with input image size $(224 \times 224)$ : Fig. 5 (a) depicts comparison of four metrics with input size $(224 \times 224)$. This figure visualizes that the proposed model outperforms existing pre-trained models in accuracy, sensitivity, and jaccard. The proposed model reports lower specificity than GoogleNet and similar performance on other models.

- Performance with input image size $(128 \times 128)$ : Fig. 5 (b) compares the results with input size $(128 \times 128)$ and the proposed model exhibits superior performance on COVID5K dataset in all metrics except sensitivity. The proposed MNRSC model fails to attain better sensitivity due to data imbalance.

ROC curve visualizes the plot between true positive rate vs false positive rate. Fig. 6 visualizes comparison of ROC curves among the proposed model and its competitive models. The existing pre-trained models report lesser performance on the COVID5K dataset as shown in Fig. 6. However, our model exhibits the best characteristics than its competitive models. 


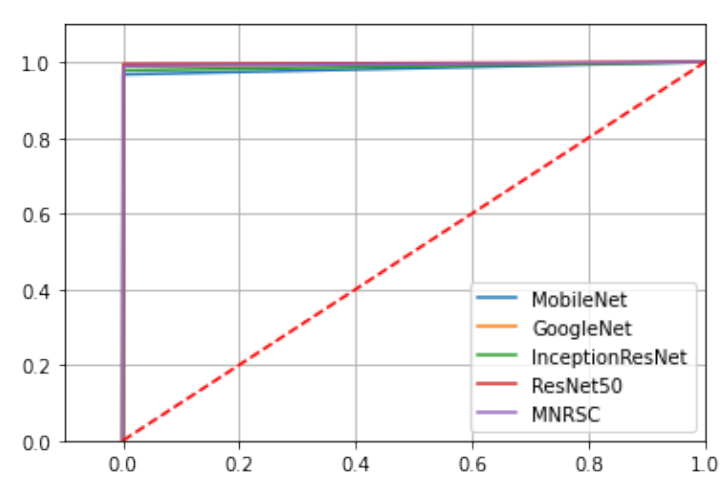

Fig. 6: Comparison of ROC curves

\subsubsection{Performance analysis on noisy data}

In general, noise is another image artifact that influences the performance of the model. Thus, we have evaluated the proposed model on noisy datasets. Medical images suffer from random noise and hence we have imposed the random Gaussian noise on the dataset. We have created a noise image having zero mean with a standard deviation of 5 . Table 5 lists out sample noise images along with their original and random noise images. If $I(w, h, c)$ is image, $R N_{\mu, \sigma}(w, h, c)$ is random noise function, then the noise image $N I(w, h, c)$ can be obtained using eq. 1.

$N I(w, h, c)=I(w, h, c)+R N_{\mu=0, \sigma=5}(w, h, c)$

Table 6 compares the results of the proposed model with its competing models on the noisy COVID5K dataset. The proposed MNRSC model has acquired second with $99.71 \%$ accuracy.

Table 6: Comparison on noisy COVID5K dataset

\begin{tabular}{|c|cccc|}
\hline Model & Accuracy & Sensitivity & Specificity & Jaccard \\
\hline GoogleNet & 99.71 & 99.71 & 99.94 & 99.43 \\
InceptionResNet & 99.17 & 99.17 & 99.84 & 98.38 \\
ResNet50 & $\mathbf{9 9 . 8 6}$ & $\mathbf{9 9 . 8 6}$ & 99.88 & $\mathbf{9 9 . 7 3}$ \\
MobileNet & 99.32 & 99.32 & $\mathbf{9 9 . 9 6}$ & 98.66 \\
MNRSC & 99.71 & 99.71 & 99.88 & 99.43 \\
\hline
\end{tabular}

\subsubsection{Empirical time complexity}

Training time is another vital factor that needs to be considered while designing effective deep networks. In general, training time mainly depends on the number of parameters and training images. In pre-trained MobileNet, the softmax layer receives a vector of size 50176 $(7 \times 7 \times 1024)$ which can be treated as neurons for decision making. A softmax layer maps these 50176 neurons into two neurons for binary classification. In the proposed model, a softmax layer receives only 512 neurons as the RSC block transforms $(7 \times 7 \times 1024)$ vector into 512 neurons. The proposed model uses $0.3 \mathrm{M}$ additional parameters than basic MobileNet. Table 7 evidence that the proposed MNRSC model outperforms its competitive models other than the MobileNet model. However, the proposed MNRSC model takes an additional training time of 4 sec. on COIVD5K than MobileNet.

Table 7: Number of parameters and training time.

\begin{tabular}{|c|c|c|}
\hline Model & \# Parameters & Training time (sec.) \\
\hline GoogleNet & $21.905 \mathrm{M}$ & 101.5 \\
InceptionResNet & $54.413 \mathrm{M}$ & 234.2 \\
ResNet50 & $23.788 \mathrm{M}$ & 118.2 \\
MobileNet & $3.329 \mathrm{M}$ & 89.9 \\
MNRSC & $3.626 \mathrm{M}$ & 93.5 \\
\hline
\end{tabular}

\subsection{Evaluation on COVIDRD dataset}

Covid radiography (COVIDRD) is another dataset published by Kaggle [18]. Table 8 lists out the cross-validation results of the proposed model on the dataset. This table reveals that the proposed model attains $100 \%$ specificity in the first four folds and $99.81 \%$ in the fifth fold. We have achieved a consistent performance of $99 \%$ for other metrics including accuracy, sensitivity, and jaccard. Fig. 7 visualizes the training and validation

Table 8: Fivefold cross-validation on COVIDRD dataset

\begin{tabular}{|c|cccc|}
\hline Fold & Accuracy & Sensitivity & Specificity & Jaccard \\
\hline 1 & 99.71 & 99.71 & 100.00 & 99.41 \\
2 & 99.80 & 99.80 & 100.00 & 99.61 \\
3 & 99.80 & 99.80 & 100.00 & 99.61 \\
4 & 99.90 & 99.90 & 100.00 & 99.80 \\
5 & 99.61 & 99.61 & 99.81 & 99.22 \\
\hline Avg. & 99.76 & 99.76 & 99.96 & 99.53 \\
Std. & 0.11 & 0.11 & 0.08 & 0.22 \\
\hline
\end{tabular}

Table 9: Sample X-ray images along with actual and predicted labels

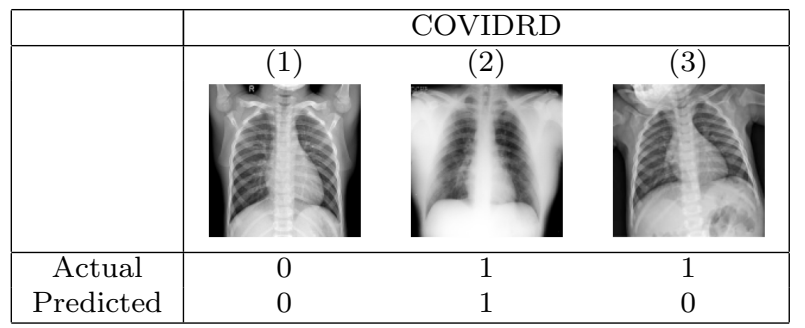

loss on COVIDRD dataset. The proposed model reports 


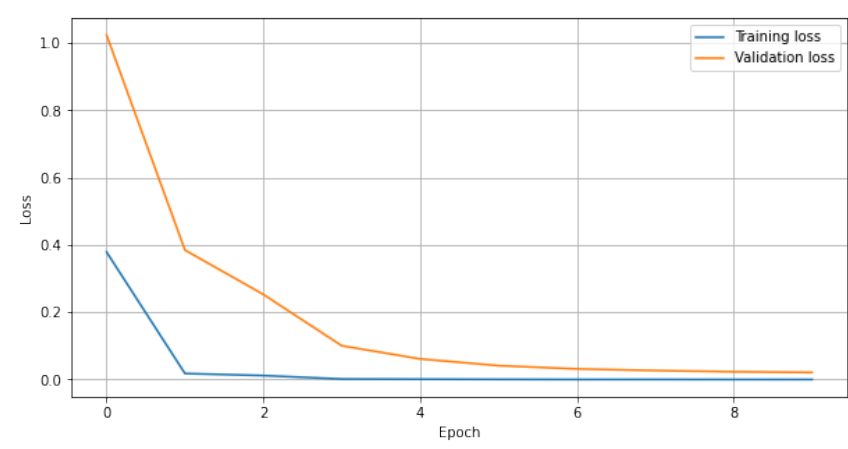

Fig. 7: Training and validation loss on COVIDRD dataset

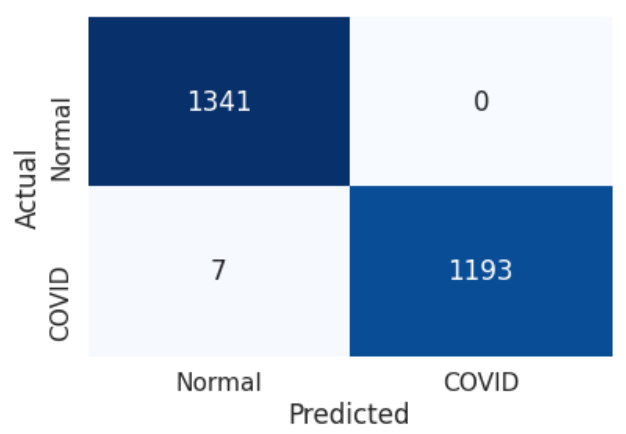

Fig. 8: Confusion matrix on COVIDRD dataset

consistent loss after sixth epoch and attains optimal loss with in ten epochs. The proposed model has classified seven COVID positive samples wrongly as negative on the COVIDRD dataset as shown in Fig. 8. However, our model has predicted all COVID negatives samples correctly. Table 9 lists out three X-ray image samples from COVIDRD dataset. Our model fails to detect dis-oriented images as shown in sample (3) of Table 9. In this image head of the patient has included due to wrong orientation.

\subsubsection{Comparison with state-of-art model}

Table 10 lists out the performance comparison with the Deep-COVID model. The Deep-COVID model attains $98.29 \%$, and $98.02 \%$ of sensitivity, and specificity of respectively. The proposed MNRSC model outperforms the existing model with $99 \%$ of sensitivity and specificity. It proves that our model achieves an improvement of $1 \%$ in both sensitivity and specificity. In medical image classification, even $1 \%$ improvement is considered as a significant performance.
Table 10: Comparison with state-of-art model

\begin{tabular}{|c|cc|}
\hline Model & Sensitivity & Specificity \\
\hline Deep-COVID & 98.29 & 98.02 \\
MNRSC & 99.76 & 99.96 \\
\hline
\end{tabular}

\subsubsection{Comparison with pre-trained models}

Performance comparison with existing pre-trained models is as follows.

- Performance with input image size $(224 \times 224)$ :

Fig. 9 (a) depicts comparison of four metrics with input size $(224 \times 224)$. This figure visualize that the proposed model outperforms existing pre-trained models in all metrics. This figures also highlight that there is a significant improvement of $7 \%$ than its backbone MobileNet in Jaccard similarity.

- Performance with input image size $(\mathbf{1 2 8} \times \mathbf{1 2 8})$ : Fig. 9 (b) compares the results with input size $(128 \times 128)$ and the proposed model exhibits superior performance on COVIDRD dataset in all metrics.

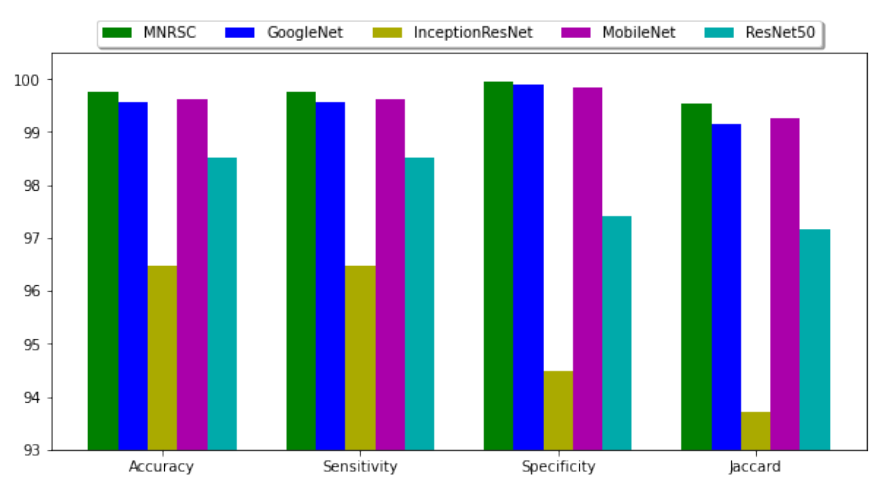

(a) input size $(224 \times 224)$

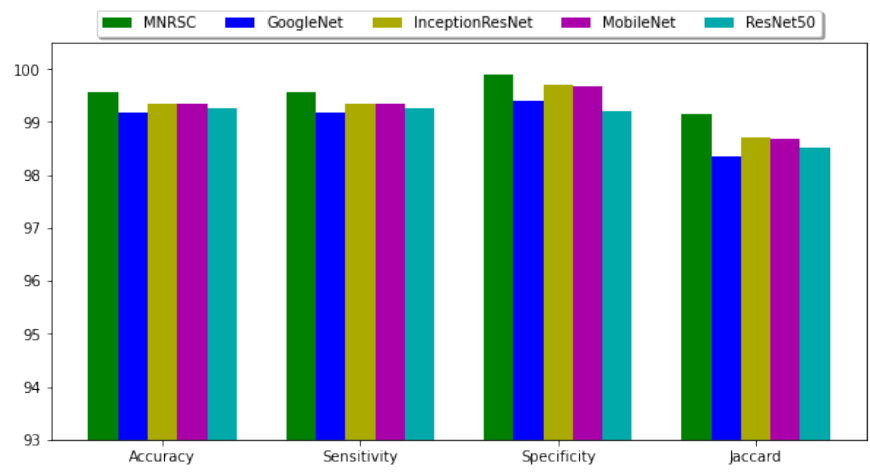

(b) input size $(128 \times 128)$

Fig. 9: Performance comparison on COVIDRD dataset

Fig. 10 visualizes comparison of ROC curves among the proposed model and its competitive models. The 
proposed model exhibits similar performance as pretrained models on the COVIDRD dataset as shown in Fig. 10.

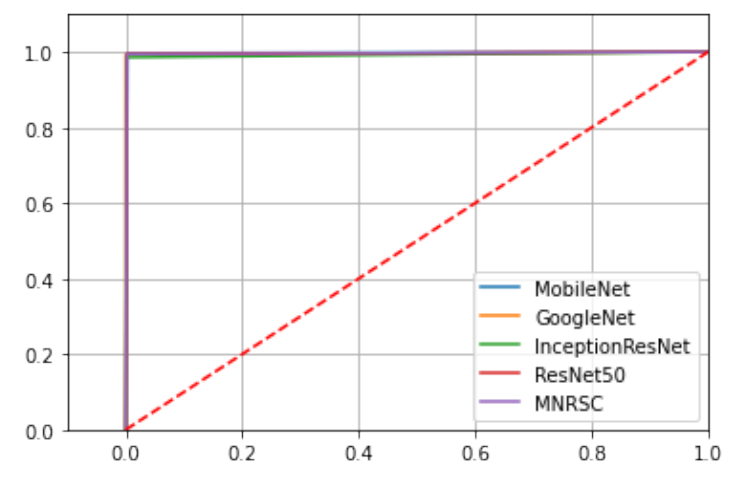

Fig. 10: Comparison of ROC curves

\subsubsection{Performance analysis on noisy datasets}

Our model outperforms its competing models on a noisy COVIDRD dataset with $99.65 \%$ accuracy and can be observed from Table 11.

Table 11: Comparison on noisy COVIDRD datasets

\begin{tabular}{|c|cccc|}
\hline Model & Accuracy & Sensitivity & Specificity & Jaccard \\
\hline GoogleNet & 99.53 & 99.53 & 99.70 & 99.06 \\
InceptionResNet & 99.61 & 99.61 & 99.70 & 99.22 \\
ResNet50 & 99.53 & 99.53 & 99.78 & 99.06 \\
MobileNet & 99.25 & 99.25 & 98.96 & 98.52 \\
MNRSC & $\mathbf{9 9 . 6 5}$ & $\mathbf{9 9 . 6 5}$ & $\mathbf{1 0 0 . 0}$ & $\mathbf{9 9 . 2 9}$ \\
\hline
\end{tabular}

\subsubsection{Empirical time complexity}

Table 12 shows that the proposed MNRSC model outperforms its competitive models other than the MobileNet model. However, the proposed MNRSC model takes an additional training time of 2 sec. on COIVDRD dataset than MobileNet.

Table 12: Number of parameters and training time.

\begin{tabular}{|c|c|c|}
\hline Model & \# Parameters & Training time (sec.) \\
\hline GoogleNet & $21.905 \mathrm{M}$ & 51.0 \\
InceptionResNet & $54.413 \mathrm{M}$ & 116.5 \\
ResNet50 & $23.788 \mathrm{M}$ & 58.9 \\
MobileNet & $3.329 \mathrm{M}$ & 44.5 \\
MNRSC & $3.626 \mathrm{M}$ & 46.7 \\
\hline
\end{tabular}

\subsection{Discussion}

We have analyzed high-level feature maps of the proposed model. Fig. 11 (a) and (b) depict convolution results after the first layer on Normal and COVID image samples respectively. These figures visualize the structural, edge, and contrast features of the images. Consider the feature map in the first-row seventh column of Fig. 11. Where the structure of the skeleton and heart has degenerated. Thus, these samples have resulted in the wrong classification. The proposed model exhibits better sensitivity on COVID5K and better specificity on COVIDRD dataset. However, the difference between sensitivity and specificity is negligible and hence our model exhibits optimal performance. Moreover, our model produces the best results on a balanced dataset like COVIDRD. On the other hand, it achieves competitive performance on imbalanced dataset like COVID5K. The proposed residual separable convolution block over MobileNet achieves significant performance on both datasets. Further, the following conclusions can be made from the above analysis.

- Our model produces better results on a balanced dataset and competitive results on an imbalanced dataset.

- Our model exhibits superior performance in accuracy, sensitivity, and Jaccard on the COVID5K dataset.

- Our model outperforms existing models in all key metrics on the COVIDRD dataset.

- It also reports consistent results with various input sizes and hence it is compatible with low-scale devices like mobiles.

- The proposed model attains a $7 \%$ improvement in jaccard similarity than MobileNet model on COVIDRD dataset.

\section{Conclusion}

COVID detection from X-ray images has become contemporary research due to increase in number of COVID cases and imbalanced datasets. However, the implementation of time-efficient models with better performance is still challenging. In this work, we have proposed a MobileNet and residual separable convolution block model for chest X-ray image classification. The proposed residual separable convolution block uses two separable convolutions, global average pooling, a dense layer, and a dropout layer. The separable convolutions with a factored residual connection have been utilized to take advantage of computational cost and feature enhancement. Global average pooling has been devised instead of Flatten layer to consider image-level features. 

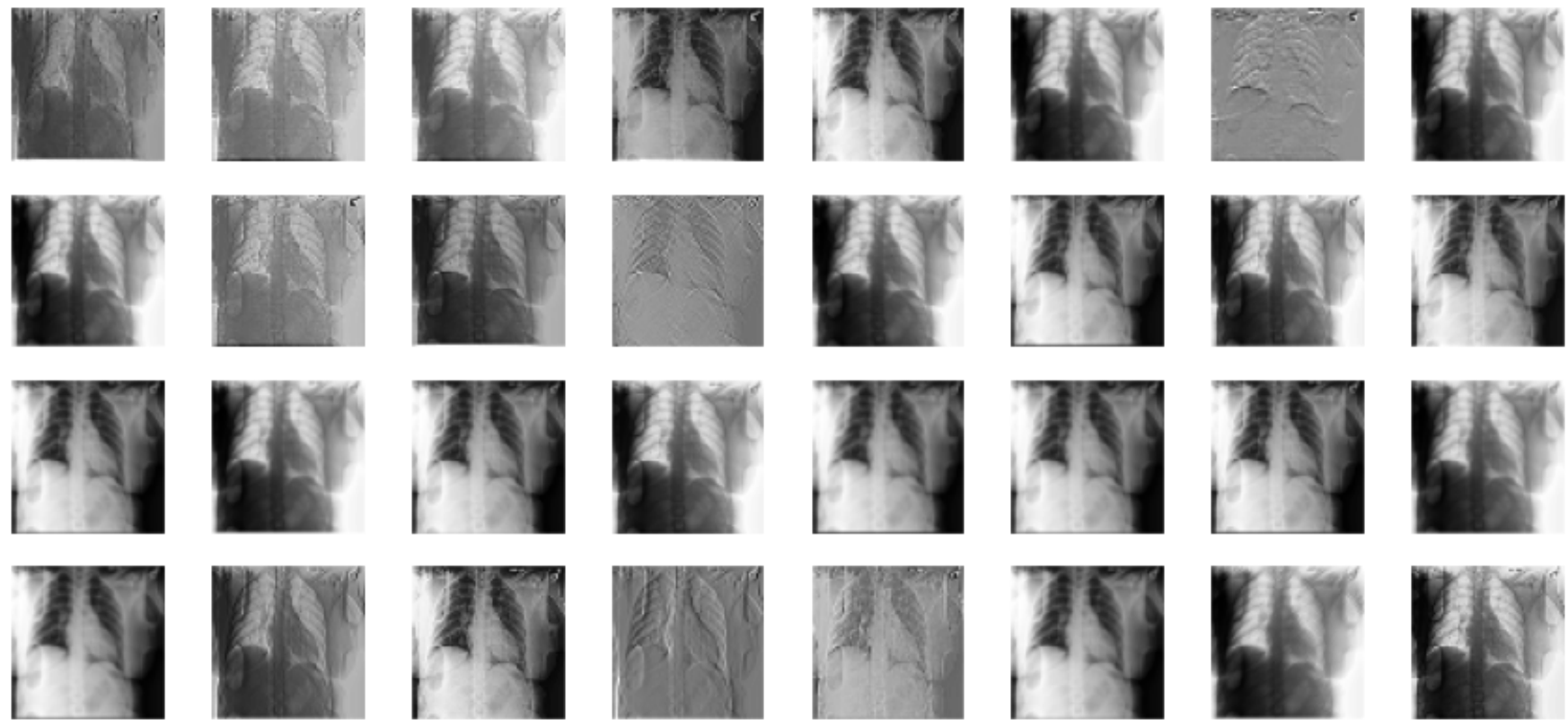

(a) Normal
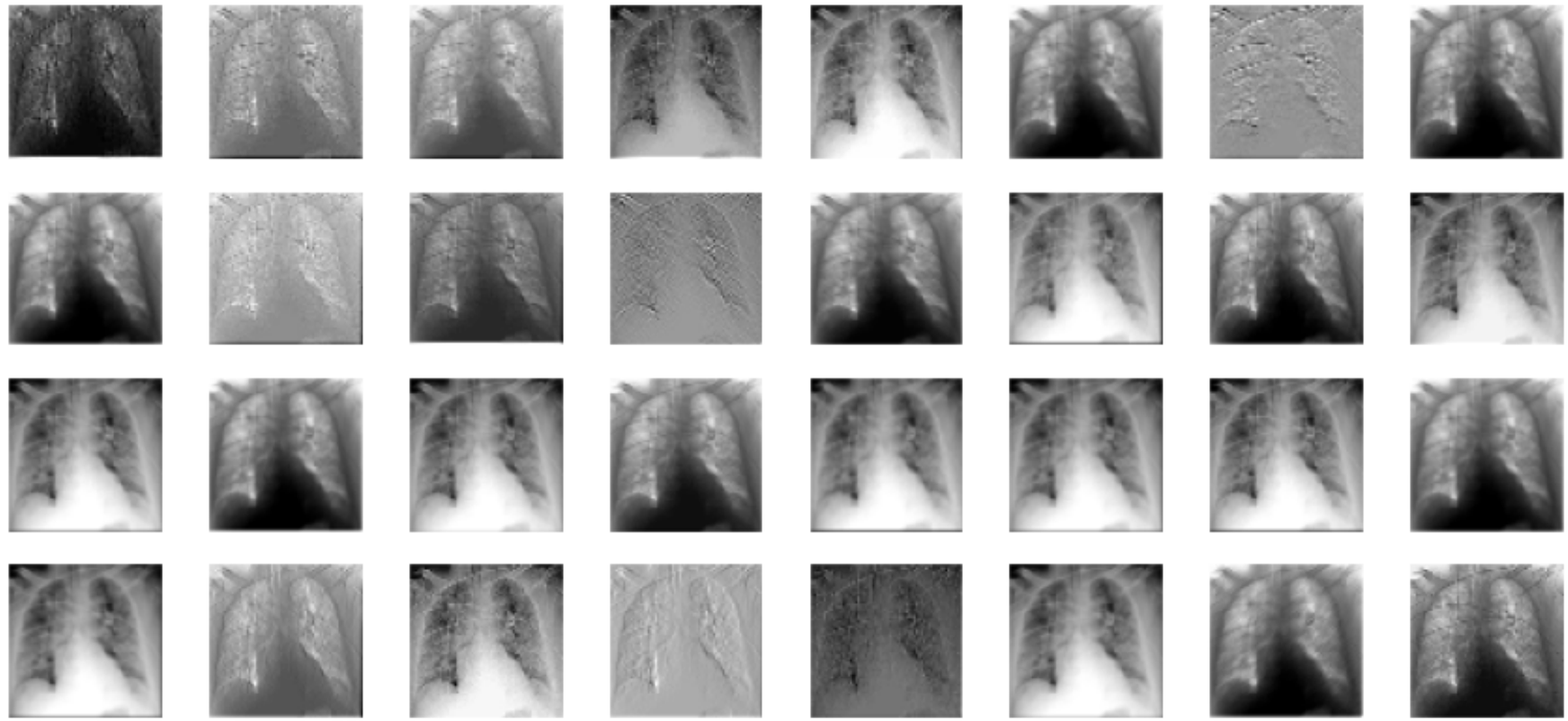

(b) COVID

Fig. 11: Convolution results at first layer

Two publicly available datasets have been considered for the performance evaluation of the proposed model. The proposed model outperforms existing pre-trained models and state-of-art models with $99 \%$ accuracy in all key metrics except specificity. However, the difference between sensitivity and specificity is negligible and hence our model exhibits optimal performance. The proposed model achieves similar results on noisy datasets.
Our proposed model incurs less training time than the existing pre-trained model and exhibits competitive performance as basic MobileNet. Further, the proposed model is compatible with mobile applications as it uses fewer parameters and lesser training time.

Compliance with ethical standards 
Conflict of interest The authors declare that there is no conflict of interest regarding the publication of this paper.

Ethical approval This article does not contain any studies with human participants or animals performed by any of the authors.

Informed consent Informed consent was obtained from all individual participants included in the study.

\section{References}

1. X. Liu, S. Zhang, Influenza and Other Respiratory Viruses (2020)

2. Weekly epidemiological update on covid-19 - 30 march 2021. URL https://www.who.int/publications/m/item/weeklyepidemiological-update-on-covid-19-31-march-2021. Accessed: 2021-04-10

3. L.A. Rousan, E. Elobeid, M. Karrar, Y. Khader, BMC Pulmonary Medicine 20(1), 1 (2020)

4. H. Liu, F. Liu, J. Li, T. Zhang, D. Wang, W. Lan, Journal of infection $\mathbf{8 0}(5)$, e7 (2020)

5. A.S. Panayides, A. Amini, N.D. Filipovic, A. Sharma, S.A. Tsaftaris, A. Young, D. Foran, N. Do, S. Golemati, T. Kurc, et al., IEEE Journal of Biomedical and Health Informatics 24(7), 1837 (2020)

6. E. Dikici, J.L. Ryu, M. Demirer, M. Bigelow, R.D. White, W. Slone, B.S. Erdal, L.M. Prevedello, IEEE journal of biomedical and health informatics 24(10), 2883 (2020)

7. T. Kodama, K. Kamata, K. Fujiwara, M. Kano, T. Yamakawa, I. Yuki, Y. Murayama, IEEE Transactions on Neural Systems and Rehabilitation Engineering 26(6), 1152 (2018)

8. M. Goyal, N.D. Reeves, A.K. Davison, S. Rajbhandari, J. Spragg, M.H. Yap, IEEE Transactions on Emerging Topics in Computational Intelligence 4(5), 728 (2018)

9. R. Ju, P. Zhou, S. Wen, W. Wei, Y. Xue, X. Huang, $\mathrm{X}$. Yang, IEEE Transactions on Emerging Topics in Computational Intelligence (2020)

10. M.Y. Ng, E.Y. Lee, J. Yang, F. Yang, X. Li, H. Wang, M.M.s. Lui, C.S.Y. Lo, B. Leung, P.L. Khong, et al., Radiology: Cardiothoracic Imaging 2(1), e200034 (2020)

11. J. Cleverley, J. Piper, M.M. Jones, bmj 370 (2020)

12. P. Afshar, S. Heidarian, F. Naderkhani, A. Oikonomou, K.N. Plataniotis, A. Mohammadi, Pattern Recognition Letters 138, 638 (2020)

13. S. Sakib, T. Tazrin, M.M. Fouda, Z.M. Fadlullah, M. Guizani, IEEE Access 8, 171575 (2020)

14. A. Abbas, M.M. Abdelsamea, M.M. Gaber, Applied Intelligence 51(2), 854 (2021)

15. S. Minaee, R. Kafieh, M. Sonka, S. Yazdani, G.J. Soufi, Medical image analysis 65, 101794 (2020)

16. M.J. Horry, S. Chakraborty, M. Paul, A. Ulhaq, B. Pradhan, M. Saha, N. Shukla, IEEE Access 8, 149808 (2020). DOI 10.1109/ACCESS.2020.3016780

17. J. De Moura, L.R. García, P.F.L. Vidal, M. Cruz, L.A. López, E.C. Lopez, J. Novo, M. Ortega, IEEE Access 8, 195594 (2020)

18. Kaggle covid-19 radiography database. URL https://www.kaggle.com/tawsifurrahman/covid19radiography-database. Accessed: 2021-01-07

19. A.G. Howard, M. Zhu, B. Chen, D. Kalenichenko, W. Wang, T. Weyand, M. Andreetto, H. Adam, arXiv preprint arXiv:1704.04861 (2017)

20. M. Lin, Q. Chen, S. Yan. Network in network (2014) 